\title{
Analysis and design of efficient coupling in photonic crystal circuits
}

\author{
P. SANCHIS ${ }^{1}{ }^{*}, \mathrm{~J}$. MART Í ${ }^{1}, \mathrm{~B}$. L U Y S SAER T ${ }^{2}, \mathrm{P} . \mathrm{DUMON}^{2}$, \\ P. BIENSTMAN ${ }^{2}$ AND R. B AETS ${ }^{2}$ \\ ${ }^{1}$ Valencia Nanophotonics Technology Center, Universidad Politécnica de Valencia, Camino de Vera s/n, \\ 46022 Valencia, Spain \\ ${ }^{2}$ Department of Information Technology, Ghent University-IMEC, Sint-Pietersnieuwstraat 41, B-9000 \\ Ghent, Belgium \\ (*author for correspondence: E-mail: pabsanki@ntc.upv.es)
}

\begin{abstract}
A rigorous analysis and design of efficient coupling from photonic crystal ( $\mathrm{PhC}$ ) waveguides into conventional dielectric waveguides is reported. Closed-form expressions for the reflection and transmission matrices that completely characterize the scattering that occurs at the interface are derived based on an eigenmode expansion technique and a Bloch basis. Analytic expressions are used to analyze the reflection into $\mathrm{PhC}$ waveguides. We obtain that negligible reflection can be achieved by choosing a certain interface within a PhC unit cell. Furthermore, analytic expressions are used to design a novel and compact coupler structure in order to achieve high coupling efficiency when broad dielectric waveguides are considered. Thereby, transmission efficiencies near $100 \%$ from the fundamental guided Bloch mode into the fundamental waveguide mode are achieved.
\end{abstract}

Key words: eigenmode expansion, electromagnetic scattering by periodic structures, mode-matching, photonic crystals

\section{Introduction}

Photonic crystals $(\mathrm{PhC})$ have been the subject of an increasing research effort in order to develop micro-scale photonic integrated circuits (Joannopoulos et al. 1995). However, efficient coupling into and out of $\mathrm{PhC}$ circuits is one of the main challenges to achieve reliable micro-scale photonic integrated circuits based on the $\mathrm{PhC}$ technology. Coupling losses between conventional dielectric waveguides and $\mathrm{PhC}$ waveguides originate from the mode mismatch between both types of waveguides. Waveguides in $\mathrm{PhC}$ circuits are usually formed by inserting line defects into the otherwise periodic structure. The creation of a line defect results in guided modes within the photonic band gap (PBG), whose propagation is determined by the Bloch theorem (Bloch 1928). On the other hand, propagation in conventional dielectric waveguides relies on index-contrast guiding. At the moment, coupling losses in $\mathrm{PhC}$ circuits have mainly been studied by means of simulations, while theoretical work remains very scarce, (Palamaru and Lalanne 2001; Botten et al. 2003; Ushida et al. 2003; Biswas et al. 2004; Sanchis et al. 2004). However, the knowledge of analytic expressions for the transmission and 
reflection at an interface between an external medium and a $\mathrm{PhC}$ circuit can be very useful when testing novel designs and studying the influence of different parameters on the coupling efficiency. Analytic expressions can dramatically reduce the computing time, by avoiding complex numerical simulations, which usually require huge computational resources. Recently, closed-form expressions based on an eigenmode expansion technique were derived for an interface between a dielectric waveguide and a semi-infinite $\mathrm{PhC}$ waveguide (Sanchis et al. 2004).

In this paper, transmission and reflection matrices are obtained for the (reverse) interface between a semi-infinite $\mathrm{PhC}$ waveguide and a dielectric waveguide as shown in Fig. 1(a). Contrasting with the previous work, in this case both forward and backward propagating Bloch modes inside the $\mathrm{PhC}$ waveguide need to be considered. Once analytic expressions are derived, they are first used to analyze the reflection into the $\mathrm{PhC}$ waveguide considering different interfaces between the dielectric and $\mathrm{PhC}$ waveguide, which are obtained by different cut positions that can be chosen within the basic period of the $\mathrm{PhC}$. We found that the cut position that provides maximum transmission efficiency is not necessarily the same that provides minimum reflection into the $\mathrm{PhC}$ waveguide. Furthermore, negligible reflection is obtained at a certain cut position for a particular $\mathrm{PhC}$ structure which can be useful to avoid Fabry-Perot resonances in the transmission spectrum of $\mathrm{PhC}$ structures of finite length. Analytic expressions are also used to analyze the transmission efficiency for different dielectric waveguide widths. We obtain that the transmission efficiency is rather low, even choosing the optimum cut position, when broad dielectric waveguides are considered. However, a novel and compact coupler is proposed in order to improve the transmission efficiency up to nearly $100 \%$. Basically, the coupler structure converts a multimode excitation with a high efficiency into a single-mode excitation of the dielectric waveguide. The limitations of the proposed coupler structure are also discussed.
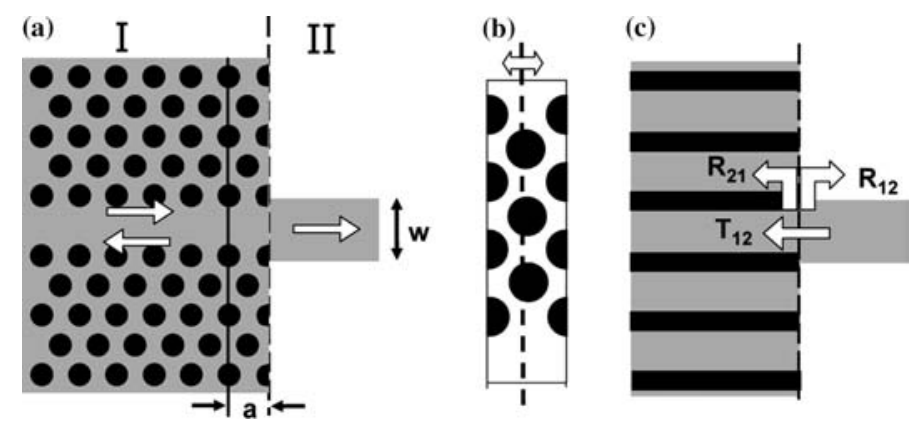

Fig. 1. (a) Analyzed structure where $w$ is the width of the dielectric waveguide and $a$ is the lattice constant, (b) supercell used to calculate the Bloch modes and (c) definition of $R_{12}, R_{21}$ and $T_{12}$. 


\section{Interface between photonic crystal and dielectric waveguides}

Figure 1(a) shows the analyzed interface formed by a line defect $\mathrm{PhC}$ waveguide butt-coupled to a dielectric waveguide. The transmission and reflection matrices are calculated by using the well known mode matching technique (Zaki et al. 1988). Therefore, we consider that an interface is placed at $z=0$ and a single Bloch mode with index $p$ is incident from medium I. This incident mode will give rise to a reflected field in medium I and a transmitted field in medium II. We expand the fields in terms of the Bloch modes in medium I and in terms of the eigenmodes in medium II. Imposing the continuity of the tangential components of the total field at the interface yields

$$
\begin{aligned}
& E_{p}^{\mathrm{I}+}+\sum_{j} R_{j, p} E_{j}^{\mathrm{I}-}=\sum_{j} T_{j, p} E_{j}^{\mathrm{II}}, \\
& H_{p}^{\mathrm{I}+}+\sum_{j} R_{j, p} E_{j}^{\mathrm{I}-}=\sum_{j} T_{j, p} H_{j}^{\mathrm{II}},
\end{aligned}
$$

where $E^{\mathrm{I}+}, H^{\mathrm{I}+}, E^{\mathrm{I}-}, H^{\mathrm{I}-}$ are the tangential electric and magnetic fields of the forward and backward propagating Bloch modes, respectively, and $T$ and $R$ are the transmission and reflection coefficients. In order to distinguish the forward propagating Bloch modes from the backward propagating Bloch modes we look at the power flux for the guided mode and at the imaginary part of the wave vector for the evanescent modes (Botten et al. 2001).

The unknown transmission and reflection coefficients are calculated by taking the right-cross product of (1) with $H_{i}^{\mathrm{II}}$ and the left-cross product of (2) with $E_{i}^{\mathrm{II}}$, which are the expansion fields of medium II. Here, $i$ is an arbitrary index. After integrating over the cross-section and by invoking the orthogonality relation in medium II, we get

$$
\begin{aligned}
& \left\langle E_{p}^{\mathrm{I}+}, H_{i}^{\mathrm{II}}\right\rangle+\sum_{j} R_{j, p}\left\langle E_{j}^{\mathrm{I}-}, H_{i}^{\mathrm{II}}\right\rangle=T_{i, p}\left\langle E_{i}^{\mathrm{II}}, H_{i}^{\mathrm{II}}\right\rangle, \\
& \left\langle E_{i}^{\mathrm{II}}, H_{p}^{\mathrm{I}+}\right\rangle+\sum_{j} R_{j, p}\left\langle E_{i}^{\mathrm{II}}, H_{j}^{\mathrm{I}-}\right\rangle=T_{i, p}\left\langle E_{i}^{\mathrm{II}}, H_{i}^{\mathrm{II}}\right\rangle,
\end{aligned}
$$

where the scalar product is defined as the following overlap integral

$$
\left\langle E_{n}, H_{m}\right\rangle=\iint_{S}\left(E_{n} \times H_{m}\right) \mathrm{u}_{z} \mathrm{~d} s .
$$


The reflection can be easily obtained by subtracting (3) and (4) yielding

$$
\left\langle E_{p}^{\mathrm{I}+}, H_{i}^{\mathrm{II}}\right\rangle-\left\langle E_{i}^{\mathrm{II}}, H_{p}^{\mathrm{I}+}\right\rangle=\sum_{j} R_{j, p}\left(\left\langle E_{j}^{\mathrm{I}-}, H_{i}^{\mathrm{II}}\right\rangle-\left\langle E_{i}^{\mathrm{II}}, H_{j}^{\mathrm{I}-}\right\rangle\right) .
$$

This equation can be simplified by decomposing the Bloch modes in terms of their forward, $F_{\mathrm{k}}$, and backward, $B_{\mathrm{k}}$, components. It should be noticed that the forward and backward components are different for the forward and backward propagating Bloch modes. Furthermore, the forward and backward components depend on the chosen cut position within the basic period of the PhC waveguide, as shown in Fig. 1(b). The chosen cut position will determine the index profile of the interface layer in medium I, shown at the left side in Fig. 1(c). Therefore, we get

$$
\begin{aligned}
\left\langle E_{j}^{\mathrm{I}+}, H_{i}^{\mathrm{II}}\right\rangle-\left\langle E_{i}^{\mathrm{II}}, H_{j}^{\mathrm{I}+}\right\rangle= & \sum_{k} F_{k}^{j+} \cdot\left(\left\langle\tilde{E}_{\mathrm{k}}, H_{i}^{\mathrm{II}}\right\rangle-\left\langle E_{i}^{\mathrm{II}}, \tilde{H}_{\mathrm{k}}\right\rangle\right) \\
& +\sum_{k} B_{k}^{j+} \cdot\left(\left\langle\tilde{E}_{\mathrm{k}}, H_{i}^{\mathrm{II}}\right\rangle+\left\langle E_{i}^{\mathrm{II}}, \tilde{H}_{\mathrm{k}}\right\rangle\right),
\end{aligned}
$$

where $\tilde{E}_{k}$ and $\tilde{H}_{k}$ are the electric and magnetic field of the interface layer. Equation (6) can be expressed with the following matrix equation

$$
\left\langle E_{j}^{\mathrm{I}+}, H_{i}^{\mathrm{II}}\right\rangle-\left\langle E_{i}^{\mathrm{II}}, H_{j}^{\mathrm{I}+}\right\rangle \equiv 2\left(R_{12} T_{12}^{-1} F_{+}+T_{12}^{-1} B_{+}\right),
$$

where $T_{12}$ and $R_{12}$ are defined as shown in Fig. 1(c). It should be noticed that these terms are the same as those used in (Sanchis et al. 2004). A similar expression can be obtained for the right-hand side of (5) so that we obtain

$$
-2\left(R_{12} T_{12}^{-1} F_{+}+T_{12}^{-1} B_{+}\right)=2\left(R_{12} T_{12}^{-1} F_{-}+T_{12}^{-1} B_{-}\right) R
$$

that results in the reflection matrix

$$
R=-\left(B_{-}-R_{21} F_{-}\right)^{-1}\left(B_{+}-R_{21} F_{+}\right)
$$

The transmission matrix is obtained by summing (3) and (4) and following a similar procedure as the one used above

$$
T=T_{12}^{-1}\left(F_{+}-R_{21} B_{+}\right)+T_{12}^{-1}\left(F_{-}-R_{21} B_{-}\right) R .
$$

Thereby, the transmission and reflection matrices calculated with (10) and (9) completely characterize the scattering that occurs at the interface between a $\mathrm{PhC}$ waveguide and a dielectric waveguide. These matrices involve the 
scattering properties of guided, radiation and evanescent modes. Thus, the transmitted and reflected power of the fundamental guided propagating Bloch mode into the $i$-mode of the dielectric waveguide is given by

$$
\begin{aligned}
& \eta_{T}=\left|T_{i, 0}\right|^{2}, \\
& \eta_{R}=\left|R_{i, 0}\right|^{2},
\end{aligned}
$$

where $T_{i, 0}$ and $R_{i, 0}$ are the $i$-row and first column of (10) and (9), respectively, and it is assumed that the modes are normalized.

\section{Low reflection into photonic crystal waveguides}

Based on reciprocity theorem, the transmission matrix that characterizes the transmission from the dielectric waveguide into the $\mathrm{PhC}$ waveguide is equal to the transposed of the transmission matrix that characterizes the transmission from the $\mathrm{PhC}$ waveguide into the dielectric waveguide. However, the reflection is usually different in both cases. Therefore, the transmission efficiency from the fundamental mode of the dielectric waveguide into the fundamental guided Bloch mode and vice versa will be the same while the reflection will be different. In this section, both types of reflection are analyzed.

The analytic results were obtained with a frequency-domain model based on a vectorial eigenmode expansion technique and a staircase approximation of the index profile (Bienstman 2001). This modeling tool CAMFR is freely available from the Internet (http://camfr.sourceforge.net). For the $\mathrm{PhC}$ waveguide, the Bloch modes were calculated from the eigenstates of the scattering matrix associated with the basic period. Afterwards, the field profiles and the forward and backward components of the Bloch modes were obtained at the chosen cut position within the basic period (Casey et al. 2004). The $\mathrm{PhC}$ structure considered is a two-dimensional triangular lattice of dielectric rods of Silicon $\left(n_{\mathrm{Si}}=3.4\right)$ surrounded by a homogeneous dielectric medium of silica $\left(n_{\mathrm{SiO}_{2}}=1.45\right)$. The radius of the rods is $0.2 a$ where $a$ is the lattice constant. The dielectric waveguide has a thickness of $0.5 \mu \mathrm{m}$ and a core of Silica surrounded by a cladding of air. The $\mathrm{PhC}$ waveguide is formed by removing a line of rods and the frequency of operation is $0.3(a / \lambda)$ for TM polarized waves.

Figure 2(a) shows the transmission efficiency as a function of the chosen cut position within the basic period calculated with (11). The transmission efficiency varies along the different cut positions due to the variation of the modal properties of the Bloch modes. It can be seen that the result shown in Fig. 2(a) is in agreement with that reported in Sanchis et al. (2004) as 

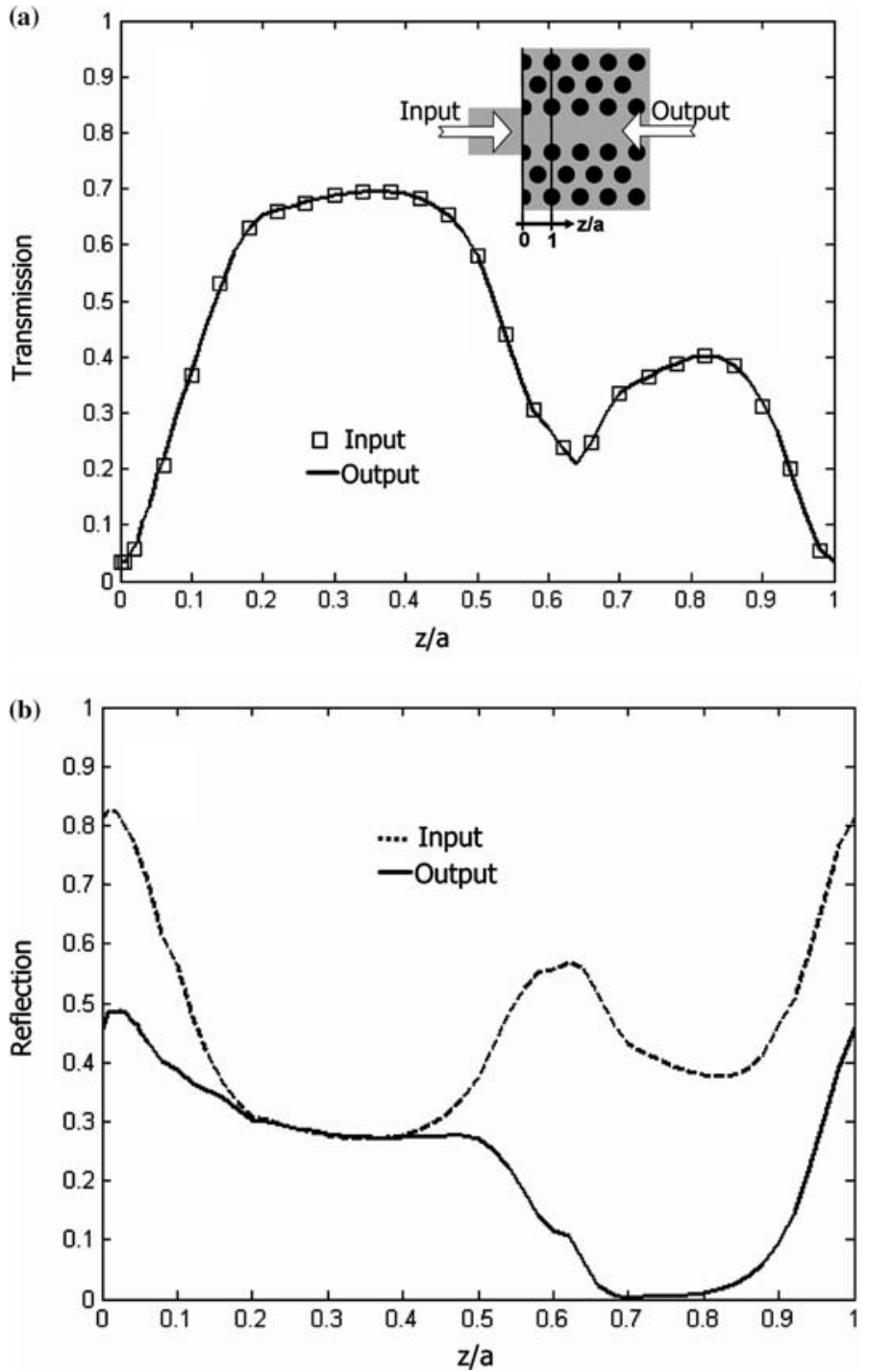

Fig. 2. (a) Transmission and (b) reflection as a function of the chosen cut position normalized by the lattice constant. The reflection is different depending on if the light is introduced or extracted from the photonic crystal.

expected from the reciprocity theorem. However, Fig. 2(b) shows the reflection as a function of the chosen cut position for two different cases: (i) input coupling where the light is propagated from the dielectric waveguide into the $\mathrm{PhC}$ waveguide and (ii) output coupling where the light is propagated from the $\mathrm{PhC}$ waveguide into the dielectric waveguide. In the former the reflection is calculated with the analytic expression derived in Sanchis et al. (2004) while in the latter it is calculated with (12). It is 
important to notice that the reflection for the input coupling case is into the dielectric waveguide while the reflection for the output coupling case is into the $\mathrm{PhC}$ waveguide. From the results shown in Fig. 2(b), it can be seen that the reflection is different for both cases except for the range of $z / a$ between 0.2 and 0.4 which corresponds with the range where the transmitted power is maximum (see Fig. 2(a)). However, the minimum reflection for the output coupling case is not achieved for the maximum transmission. The former is obtained at $z / a=0.7$ while the latter is obtained at $z / a=0.3$.

Figure 3 shows the reflection spectra for the input and output coupling cases at the cut positions which give the minimum reflection into the $\mathrm{PhC}$ waveguide $(z / a=0.7)$ and the maximum transmission efficiency $(z / a=0.3)$. The transmission efficiency at $z / a=0.3$ is around $70 \%$ while the reflection into the dielectric waveguide (input coupling) is around 30\%. Therefore, coupling losses are mainly due to reflection, while scattering losses are negligible. Scattering losses are due to the coupling to radiation modes which can only be excited in the dielectric waveguide. Therefore, it can be expected that radiation modes will also not be excited at the output coupling which could explain the similarity of the reflection spectra between both input and output coupling. However, a higher mode mismatch occurs at $z / a=0.7$ which significantly increases the coupling to radiation modes, i.e. scattering losses. In this case, the transmission is around $30 \%$ while the reflection into the dielectric waveguide is around $40 \%$ so that scattering losses will be around $30 \%$. Therefore, a high coupling to radiation modes can be expected for the output coupling, which could explain the very low reflection achieved into the $\mathrm{PhC}$ waveguide.

The minimization of the reflection that occurs when the light is extracted from the PhC waveguide can be useful to avoid Fabry-Perot resonances in the transmission spectrum of $\mathrm{PhC}$ structures of finite length. However, the transmission efficiency is reduced so a trade-off between maximum transmission and low reflection will exist. Figure 4 shows the transmission efficiency as a function of the normalized frequency for $z / a=0.3$ and $z / a=0.7$ and considering a semi-infinite $\mathrm{PhC}$ waveguide. Figure 5(a) shows the examined $\mathrm{PhC}$ structure of finite length where the optimum cut positions must be designed in both input and output interfaces. The transmission efficiency as a function of the normalized frequency for this $\mathrm{PhC}$ structure is shown in Fig. 5(b) for different cut positions at both input and output interfaces. The transmission spectra were calculated by means of FDTD simulations (Taflove 1995). An incident pulsed field was launched at an input dielectric waveguide so that the transmission spectrum was calculated with the overlap integral between the launched and measured field at the input and output dielectric waveguides, respectively.

When the input and output interfaces are chosen to achieve the maximum transmission efficiency $\left(z / a_{\text {in }}=z / a_{\text {out }}=0.3\right)$, notable Fabry-Perot resonances appear in the transmission spectrum as the reflection into the $\mathrm{PhC}$ waveguide 

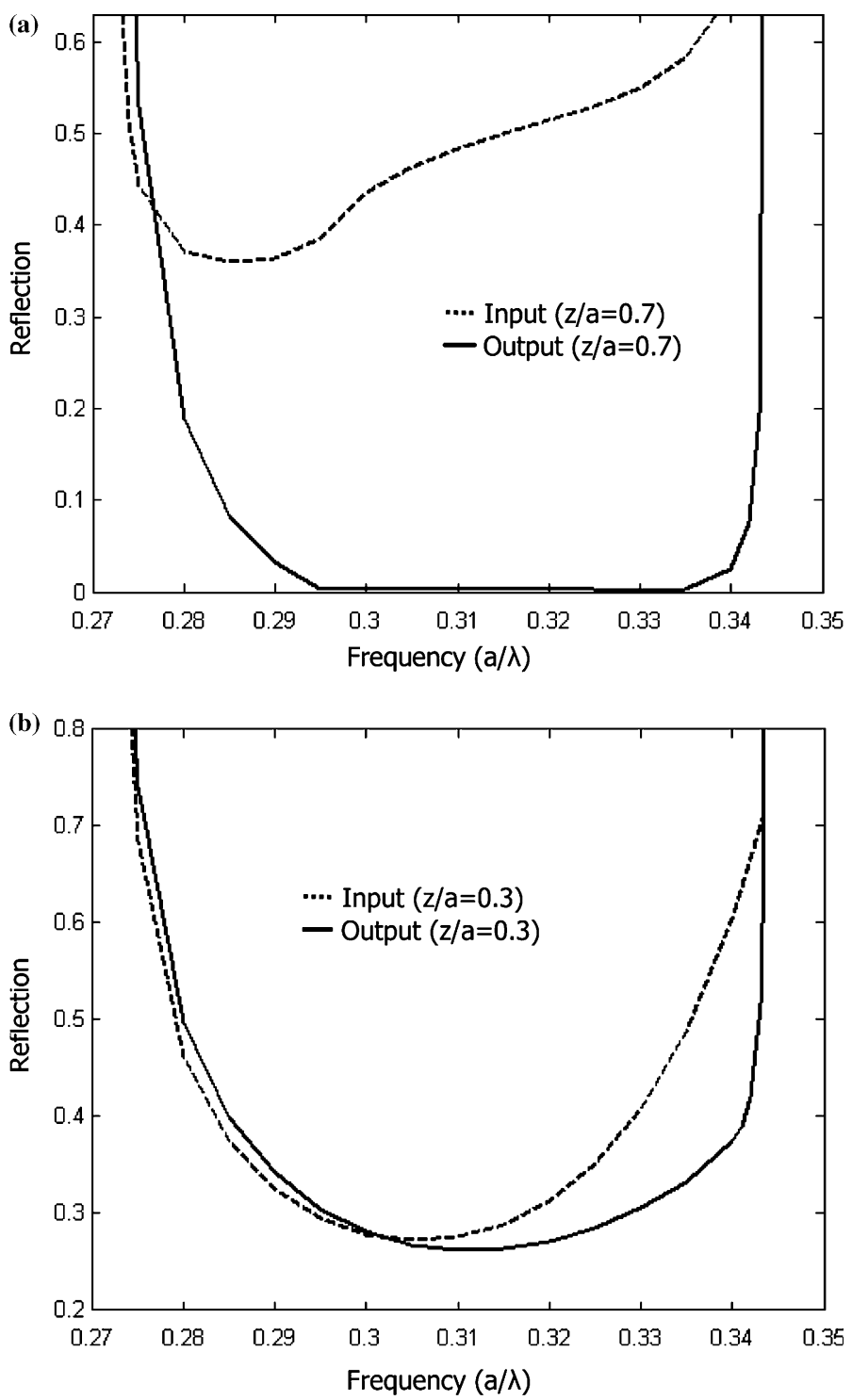

Fig. 3. Reflection as a function of the normalized frequency for the input and output coupling at (a) $z / a=0.7$ and (b) $z / a=0.3$.

is significant. It can be seen that the transmission at the resonance peaks is near unity at the frequencies where scattering is negligible. In the second case, the input and output interfaces are chosen to minimize the reflection into the $\mathrm{PhC}$ waveguide $\left(z / a_{\text {in }}=z / a_{\text {out }}=0.7\right)$. In this case, it can be seen that the Fabry-Perot resonances are eliminated but the overall transmission efficiency is very low. One possible approach to improve the transmission efficiency 


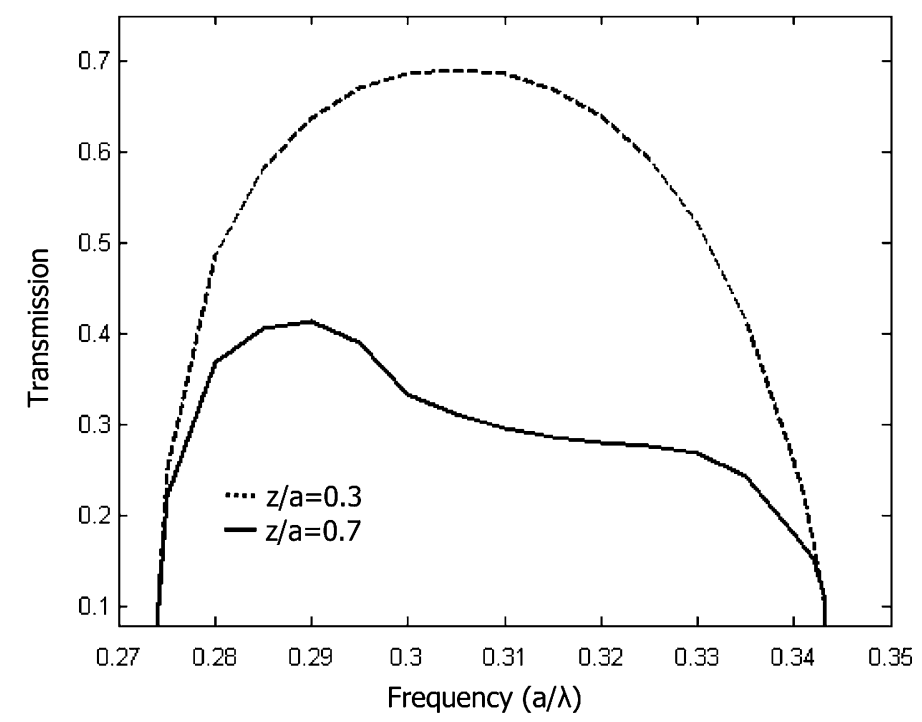

Fig. 4. Transmission efficiency as a function of the normalized frequency for different cut positions.

without increasing Fabry-Perot resonances is to use at the output interface the cut position which gives minimum reflection into the $\mathrm{PhC}$ waveguide $(z)$ $\left.a_{\text {out }}=0.7\right)$ and use at the input interface the cut position which gives maximum transmission $\left(z / a_{\text {in }}=0.3\right)$. Thus, the transmission efficiency is improved without increasing the Fabry-Perot peaks as the reflection at the output interface is still negligible. However, it can be seen that some resonances appear at the frequency range between $0.275(\mathrm{a} / \lambda)$ and $0.3(\mathrm{a} / \lambda)$ which is in agreement with the increase in reflection that can be seen in Fig. 3(a). Finally, we want to remark that the agreement between FDTD results and the analytic results for the corresponding semi-infinite $\mathrm{PhC}$ structure demonstrate the validity of the analytic expressions derived in the previous section.

\section{High transmission efficiency into broad dielectric waveguides}

The transmission efficiency decreases as broader dielectric waveguides are used due to the higher mode mismatch which mainly stems from the mode profile mismatch. Figure 6(a) shows the transmission into the fundamental mode of the dielectric waveguide as a function of the chosen cut position for three different dielectric waveguide widths: $0.5,1.5$ and $2 \mu \mathrm{m}$. In this case, a $\mathrm{PhC}$ structure formed by air holes in a dielectric medium of Silicon has been considered. The dielectric waveguide also has a core of Silicon surrounding by an air cladding. We use the effective index approximation $\left(\mathrm{n}_{\mathrm{eff}}=2.7\right)$ and a hole radius of $0.3 a$. The $\mathrm{PhC}$ waveguide is formed by removing a line of holes and the frequency of operation is $0.3(a / \lambda)$ for TE polarized waves. 
(a)
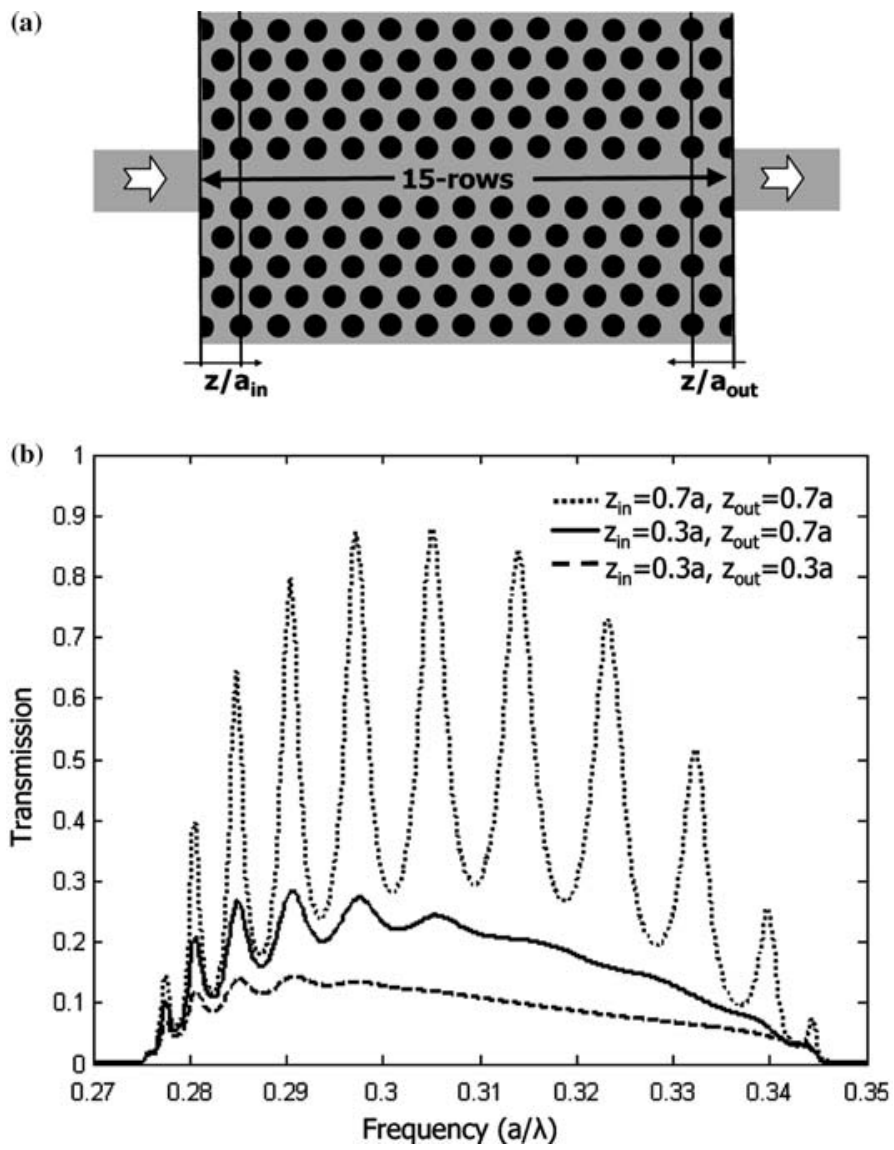

Fig. 5. (a) PhC structure of finite length considered and (b) corresponding transmission efficiency as a function of the normalized frequency calculated by means of FDTD simulations.

In Fig. 6(a), it can be seen that although high transmission can be achieved with this kind of $\mathrm{PhC}$ structure when narrow dielectric waveguides are used, the transmission significantly decreases when broader dielectric waveguides are considered even by choosing the optimum cut position. For a width of $2 \mu \mathrm{m}$, the maximum transmission efficiency is only of $67 \%$ for the optimal cut position $(z / a=0.3)$. However, the reflection into the $\mathrm{PhC}$ waveguide, shown in Fig. 6(b), is maintained below $1 \%$ even when broad dielectric waveguides are considered. This means that almost all the power is transmitted out of the $\mathrm{PhC}$ waveguide. However, the low transmission into the fundamental mode of the dielectric waveguide indicates that higher transmission occurs to the higher order guided modes as well as to radiation modes which can both be excited in the dielectric waveguide. It is important to notice that the number of guided modes that the dielectric waveguide supports increases as the waveguide width increases. For the waveguide 

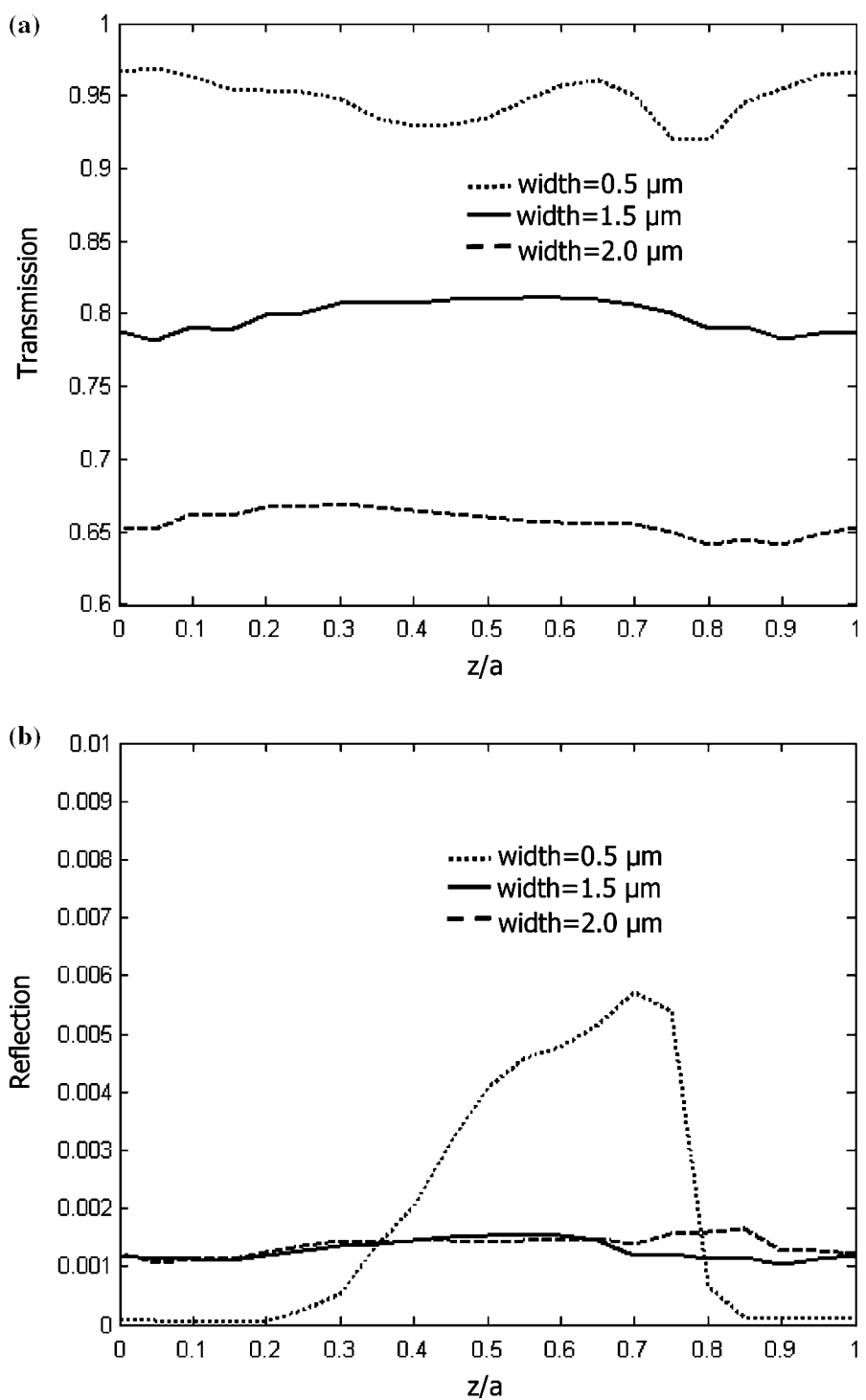

Fig. 6. (a) Transmission and (b) reflection into the $\mathrm{PhC}$ waveguide as a function of the chosen cut position and for different dielectric waveguide widths. The PhC structure in this case is formed by air holes in a dielectric background.

widths shown in Fig. 6, only the narrower waveguide with a width of $0.5 \mu \mathrm{m}$ has one even guided mode. However, three and four even guided modes can be excited in the 1.5 and $2 \mu \mathrm{m}$-wide waveguides, respectively.

There are several approaches to increase the transmitted power into and out of $\mathrm{PhC}$ waveguides using broad dielectric waveguides. However, they can be roughly separated in two groups which differ on if the $\mathrm{PhC}$ structure is 
altered at the interface, for instance using PhC tapers (see e.g. Sanchis et al. 2002) or if the dielectric waveguide structure is altered at the interface. In the latter, the simpler approach is to use the widely studied dielectric tapers (see e.g. Murphy 1988) in which the width of a broader waveguide is adiabatically varied up to a narrower width. In this case, the narrower width will be chosen in order to achieve the maximum coupling efficiency into the PhC structure. However, this approach requires long structures to obtain high transmission. For the PhC structure considered, a $5 \mu \mathrm{m}$-long linear taper was firstly used to couple a $2 \mu \mathrm{m}$-wide waveguide with the $\mathrm{PhC}$ waveguide. The linear taper reduces the waveguide width from 2 to $0.5 \mu \mathrm{m}$. Furthermore, the optimum cut position which gives maximum transmission, $z / a=0.95$, has been chosen to couple the $0.5 \mu \mathrm{m}$-wide dielectric waveguide with the $\mathrm{PhC}$ waveguide. Figure 7 shows the magnetic field diagram for the long taper used for coupling into and out a $\mathrm{PhC}$ waveguide of finite length. In this case, a transmission of $95 \%$ for the fundamental mode is achieved from the input to the ouput dielectric waveguide. However the transmission is drastically reduced to $57 \%$ when a $1.4 \mu \mathrm{m}$-long linear taper is used. Figure 8 shows the magnetic field diagram when the short taper is used.

A novel and compact coupler is proposed to improve the coupling. Figure 9 shows the magnetic field diagram for the designed coupler structure used to couple light into and out the same $\mathrm{PhC}$ waveguide of finite length. The coupler length is $1.4 \mu \mathrm{m}$. The procedure to design the coupler was the following. Firstly, the amplitude and phase of the transmission coefficients corresponding to the guided modes of the dielectric waveguide were calculated with (10) at the optimum cut position $(z / a=0.3)$. Then, the coupler structure was designed, regardless of the $\mathrm{PhC}$ structure, by means of a genetic algorithm (Michalewicz and Fosel 1998) in order to convert the multimode excitation into a single-mode excitation. The coupler consists of 14 sections with a length of $0.1 \mu \mathrm{m}$. The widths of these sections form the input of the optimization algorithm. A transmission efficiency of $98 \%$ for the fundamental mode is achieved from the input to the output dielectric waveguide. The obtained coupler structure shows very small wing-like features, in which

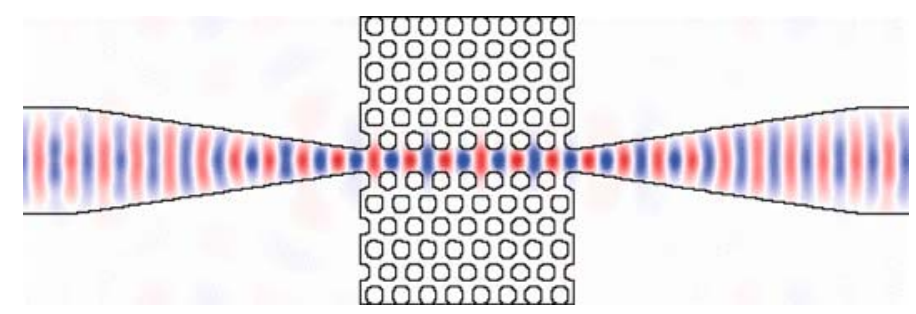

Fig. 7. Magnetic field diagram for the $5 \mu \mathrm{m}$-long linear used for coupling into and out a $\mathrm{PhC}$ waveguide of finite length using a $2 \mu \mathrm{m}$-wide dielectric waveguide. 


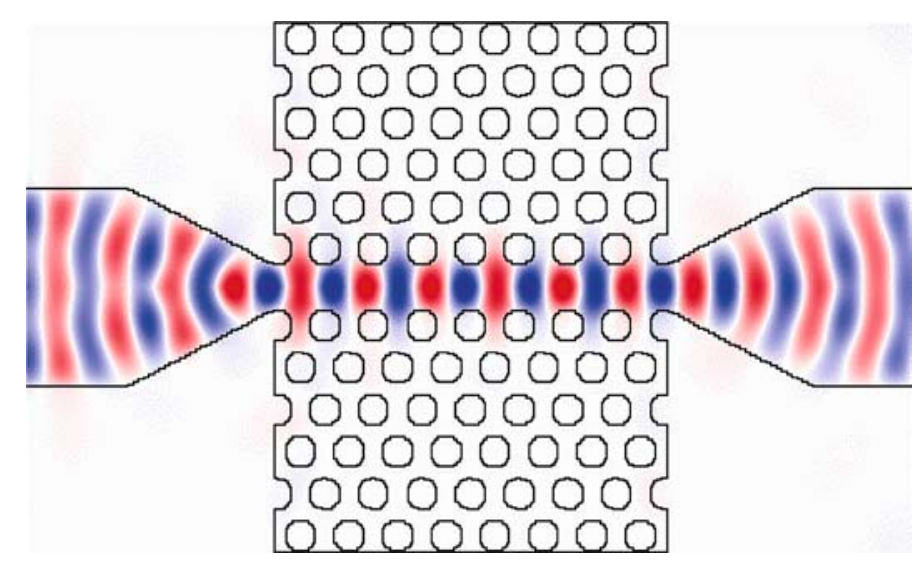

Fig. 8. Magnetic field diagram for the $1.4 \mu \mathrm{m}$-long linear used for coupling into and out a $\mathrm{PhC}$ waveguide of finite length using $2 \mu \mathrm{m}$-wide dielectric waveguide.

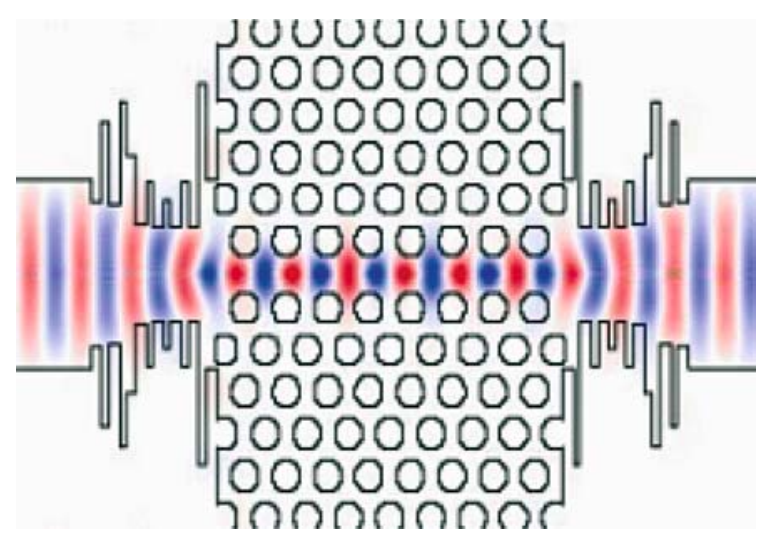

Fig. 9. Magnetic field diagram for the $1.4 \mu \mathrm{m}$-long compact coupler used for coupling into and out a $\mathrm{PhC}$ waveguide of finite length using a $2 \mu \mathrm{m}$-wide dielectric waveguide.

the magnetic field has a small value. Although their exact role has not yet been determined, they are necessary for the structure to function well. When the three middle wing-like waveguide sections are replaced by sections with a width that is the average width of the two neighbouring sections, the overall transmission efficiency of the structure drops below $80 \%$. Figure 10 shows the butt coupling case in which a transmission efficiency of only $41 \%$ is achieved, even using the optimum cut position. Furthermore, it can be seen that the magnetic field has a complex pattern due to the high reflection and interaction between the multiple guided modes.

We also tried to repeat a similar design for coupling to the rod $\mathrm{PhC}$ structure considered in Section 3 using a broader dielectric waveguide. The problem was that in this case most of the power was scattered into radiation 


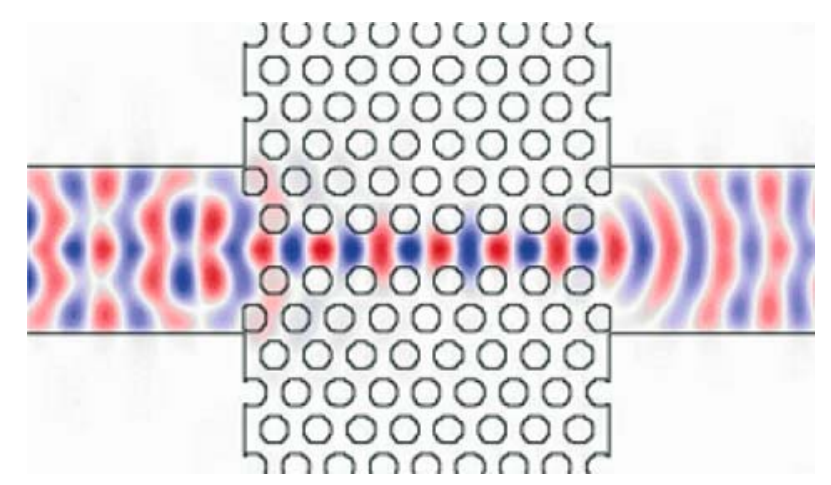

Fig. 10. Magnetic field diagram for the butt-coupling between a $2 \mu \mathrm{m}$-wide dielectric waveguide and the $\mathrm{PhC}$ waveguide of finite length.

modes mainly due to the low index contrast of the dielectric waveguide. Therefore, the transmitted power was not significantly improved regarding the butt-coupling structure. In conclusion, high transmission efficiency is achieved with the proposed coupler structure when almost all the power is scattered into the guided modes of the dielectric waveguide that means that the reflection into the $\mathrm{PhC}$ waveguide has to be very low as well as the coupling to the radiation modes which can be excited in the dielectric waveguide. In this context, the obtained analytic expressions are a useful instrument for efficiently testing different dielectric and $\mathrm{PhC}$ structures avoiding complex and huge time consuming simulations.

\section{Conclusions}

Closed-form expressions for the reflection and transmission matrices at the interface between a $\mathrm{PhC}$ structure and an external medium have been derived. The obtained formulas in addition to those obtained in Sanchis et al. (2004) can be very useful for completely analyzing the influence of different parameters on the coupling efficiency as well as for efficiently helping in the design of novel structures.

We have also shown that the reflection into a $\mathrm{PhC}$ waveguide can be minimized by using the appropriate cut position chosen within the basic period of the PhC. Furthermore, a compact coupler structure has been proposed in order to improve the coupling efficiency when broad dielectric waveguides are used. The proposed coupler structure converts a multi-mode excitation into a single-mode excitation. Therefore, transmission efficiencies near $100 \%$ from the fundamental guided Bloch mode into the fundamental mode of the dielectric waveguide can be achieved. 
Finally, we want to point out that only two-dimensional problems have been considered here. However, analytic expressions can also be used for three-dimensional (3D) problems in which the advantages when comparing with conventional numerical simulations, such as FDTD, will be much more noteworthy. In this case, only the $3 \mathrm{D}$ calculation of the Bloch modes of the corresponding $\mathrm{PhC}$ waveguide as well as the $3 \mathrm{D}$ calculation of the dielectric waveguide modes is required in order to use analytic expressions.

\section{Acknowledgements}

This work has been partially funded by the Spanish Ministry of Science and Technology under grant TIC2002-01553. Parts of this work were also performed in the context of the Belgian DWTC project IAP-Photon. P. Sanchis acknowledges the Spanish Ministry of Education, Culture and Sport for funding his grant. P. Bienstman acknowledges the Flemish Fund for Scientific Research (FWO-Vlaanderen) for a postdoctoral fellowship. B. Luyssaert and P. Dumon acknowledge the Flemish Institute for the Industrial Advancement of Scientific and Technological Research (IWT) for a specialization grant.

\section{References}

Bloch, F. Z. Physik. 52 555, 1928.

Bienstman, P. Ph.D. dissertation, Ghent University, Belgium, 2001.

Biswas, R., Z.Y. Li and K.M. Ho. Appl. Phys. Lett. 84(8) 1254, 2004.

Botten, L.C., N.A. Nicorovici, R.C. McPhedran, C. Martijn de Sterke and A.A. Asatryan. Phys. Rev. E 64 046603, 2001.

Botten, L.C., A.A. Asatryan, T.N. Langtry, T.P. White, C. Martijn de Sterke and R.C. McPhedran. Opt. Lett. 28(10) 854, 2003.

Casey, K.H., E. Lidorikis, X. Jiang, J.D. Joannopoulus, K.A. Nelson, P. Bienstman and S. Fan. Phys. Rev. B $69195111,2004$.

Joannopoulos, J.D., R.D. Meade and J.N. Winn. Photonic Crystals. Princeton University Press, Princeton, NJ, 1995.

Michalewicz, Z. and D.B. Fogel. How to solve it: Modern Heuristics, Springer, Berlin, 1998.

Murphy, E.J. IEEE J. Light. Tech. 6 862, 1998.

Palamaru, M. and P. Lalanne, Appl. Phys. Lett. 78 1466, 2001.

Sanchis, P., J. Martí, J. Blasco, A. Martínez, and A. García. Opt. Express 10 1391, 2002.

Sanchis, P., P. Bienstman, B. Luyssaert, R. Baets and J. Marti. IEEE J. of Quantum Electro. 40(5) 541, 2004

Taflove, A. Computational Electrodynamics, Norwood, MA, Artech, 1995.

Ushida, J., M. Tokushima, M. Shirane, A. Gomyo and H. Yamada. Phys. Rev. B 68 155115, 2003.

Zaki, K.A., S. Chen, and C. Chen. IEEE Trans. Microwave Theor. Tech. 36 1804, 1988. 